\title{
Electronic-Nose System for Classification of Fruits and Freshness Measurement using K-NN Algorithm
}

\author{
S.Kalpana, A.Lakshmi Baghyam
}

\begin{abstract}
The main objective of this work is to design electronic nose system (E-Nose) which detects the odour and freshness of different fruits such as mango, pineapple, orange which are mainly used in food manufacturing industries. E-Nose system consists of sensor array of two with each sensor respond to different types of odours. These sensor data is analyzed with the K-nearest neighbour algorithm (K-NN Algorithm) using MATLAB for identification of different fruits. Freshness of fruit juice is determined by the measurement of $\mathrm{pH}$ value of juice by using $\mathrm{pH}$ electrode.
\end{abstract}

Index Terms- Electronic nose, odours, $\mathrm{KNN}$ algorithm, $p \mathrm{H}$ value.

\section{INTRODUCTION}

When the students visited the food industry where their outcomes are jam production, fruits packing there are many methods for separation of fruits and also manual process [1].Electronic-nose system has used for the identification, classification of different fruits. Electronic noses are been designed as automated system for the detection and classification of odours, vapours, and gases. The main objective of this study is to design electronic nose system which detects the odour of the fruits such as mango, pineapple and orange which are mainly used in food manufacturing industries. E-Nose system consists of sensors which respond to a different type of odours i.e. alcohol content and ammonia in a fruit. E-nose system has great involvement for the identification, classification of different fruits

\section{EXISTING WORK}

Some few methods that is existing for fruit classificationwhich are discussed as follow, the standard approach to odour analysis is to use analytical chemistry instruments like Gas Chromatography and Mass Spectrometry (GC/MS) [2]. These ways are good in identifying smells; however they are time overwhelming and high cost. This GC/MS technique is a time-consuming technique and because of this disadvantage E-nose has developed by using ANN (artificial neural network) algorithm [3] in order to train the system two types of ANNs were created.in that two ANNs one type isstandard multilayer feed-forward network trained with the backpropagation algorithm and the second one isfuzzy ART map algorithm During the system operation a chemical gas

Revised Manuscript Received on April 12, 2019.

S.Kalpana,Dept of EIE, V.R.Siddhartha Engineering College, Vijayawada.A.P, India.

A.LakshmiBaghyam, Dept of EIE, V.R.Siddhartha Engineering College, Vijayawada.A.P, India. vapouris spreadacross the sensor array, the sensor outputs are digitized and fed into the computer, and the ANN (implemented in LabVIEW) then identifies the chemical gas. Time taken to identify the chemical is dependent on sensor response time which is on the order of seconds only used for small household purpose applications and due to these disadvantages identification of fruits k-NN algorithm [4] is developed.

\section{PROPOSED SYSTEM}

The Fig. 1 represents the block diagram of Electronic-nose system for classification of fruits and freshness measurement.

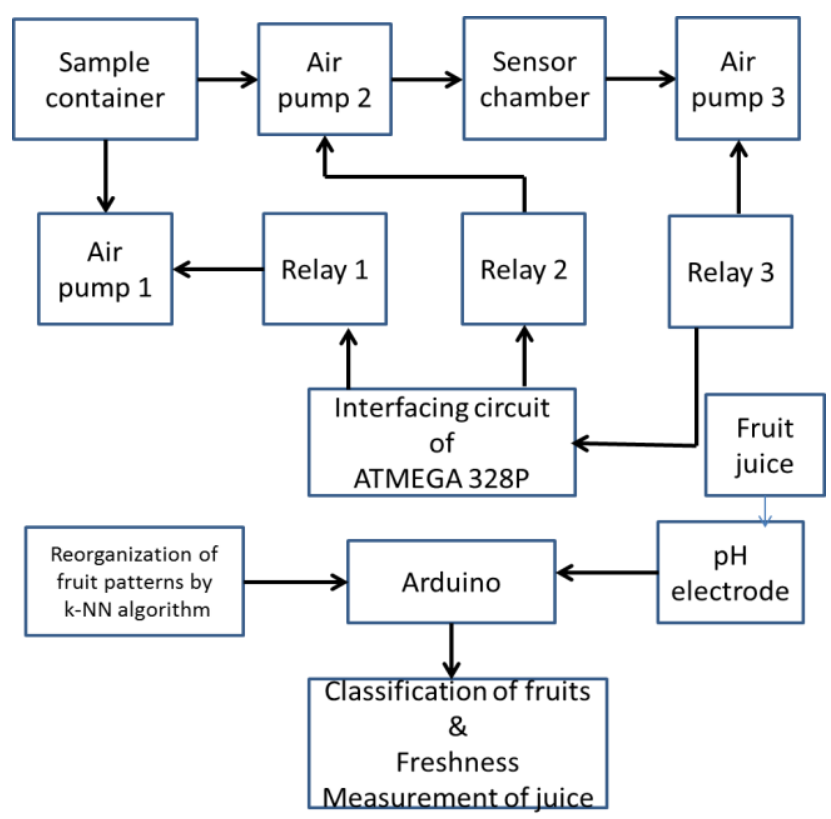

Fig.1. Block diagram of E-Nose System

Different fruits are taken such as orange, pineapple and mango and placed in the sample container. Firstly, the air present in the sensor chamber must empty. Then by pressure pump motor the air is sent into the sample container where the fruit slice is present, the gas which enters into the chamber mixes with the gas which is evolved from the fruit is then send to the sensor chamber. In the sensor chamber the gas sensors such as MQ-3[5] and MQ-135[6] are present which detects the alcohol and ammonia content respectively, which are present in the gas (by setting limit). By taking this sample values for number of times we can create a dataset to

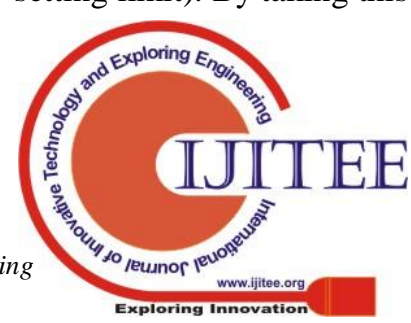


train the system by using k-NN algorithm in MATLAB. And also a $\mathrm{pH}$ sensor [7] is included in the sensor chamber by which the $\mathrm{pH}$ of different fruits can be determined (expressed in terms of $\mathrm{pH}$ value) which gives the freshness of the fruit in terms of acidity.

\section{A. Sample Container}

A plastic container is made of high density plastic. Sample plastic container is taken for placing the fruit samples such as orange, pineapple and mango in it.

\section{B. Sensor Chamber}

The sensor chamber is a closed loop area that allows gas flow to ensure maximum contact with the sensors array for accurate gas detection and measurements of different gas parameters. The sensors are placed inside it for the odour detection of the fruit. The sensors used in the sensor chamber are MQ-3 sensor and MQ-135 sensor. An analog $\mathrm{pH}$ meter is used for the detection of amount of $\mathrm{pH}$ contained in a fruit juice.

The input voltage for MQ-3 sensor is 5v. The MQ-3 sensor consists of 4 pins A0, D0, GND and VCC. In the four pins $\mathrm{A} 0$ is connected to Arduino analog A0 pin, the GND pin is connected to the GND in, VCC is connected to $5 \mathrm{v}$ VCC pin in Arduino board. The MQ-3 sensor range for orange is given as $<100$, for mango it is in between $>100$ to $<120$ and for pineapple it is in between $>120$ to $<140$.

The input voltage for MQ-135 sensor is 5v. The MQ-135 sensor consists of 4 pins a0, d0, GND and VCC. The VCC pin of MQ-135 is connected to the Arduino 5v pin, the ground pin is connected to the GNDpin and a0 pin is connected to the analog input pin A1 of the Arduino board. the mq-135 sensor range for orange is given as $>540$, for mango it is in between $>525$ to $<540$ and for pineapple it is in between $>510$ to $<525$.

The $\mathrm{pH}$ sensor can interfaced with a micro-controller, such as Arduino. The A5 pin ofArduino is connected to P0 pin of the sensor module and VCC - GND pins are connected to the VCC - GND pins of sensor module. The $\mathrm{pH}$ range of mango ranges from $3.60-4.30$ [8], $\mathrm{pH}$ range of pineapple ranges from $0.50-0.80$ [9] and $\mathrm{pH}$ range of orange ranges from $1.50-2.50[10]$.

\section{Air Pump Motor}

The air pump consists of two terminals, positive and ground. Positive pins are connected to $\mathrm{NC}$ pin of relay and ground is connected to COM of relay. An air pump is used for pushing air into containers . These air pumps contains piston, vane, impeller and diaphragm for their operation. Here a mini 6 Volt D.C air pump is used.

\section{Relay Unit}

The input IN pin is connected to the motor. The GND of all relays are connected to common ground. $5 \mathrm{~V}$ relay signal input voltage range $0-5 \mathrm{~V}$. in this work relays are used to switch on the air pump motors to pump the fresh air into the container and sensor chamber .

\section{E. ATMEGA328P}

The Arduino UNO ATmega328P consists of analog pins A0 - A5, digital pins $0-13$. The input supply of the ATmega328P microcontroller is $5 \mathrm{v}$. The sensors output is connected to the analog pins (MQ-3 to pin A0 and MQ-135 to pin A1). This analog data is then converted into digital data by using an A/D converter. The three relay devices used are connected to the Arduino board to the pins 8,9,10 respectively. The ground pins GND of Arduino are connected to the GND pins of sensors and relays.

Arduino board is used as interfacing device between hardware and software. It is used to convert the analog sensor data into digital data by using $\mathrm{A} / \mathrm{D}$ converter.

\section{F. Training Data}

Training has been given to the e-nose system by taking number of iteration data of the fruit. The data is taken for three days with respect to three different fruits for three times a day. The main aim of this training process is to configure the identification system to produce unique classification. This training data can be later given for the fruit identification or classification purpose.

\section{G. Fruit Classification}

The odour can be classified based on the amount of alcohol content present in each fruit such as mango, pineapple and orange. The input of the odour classifier can be obtained from the trained data of the fruit. By using the KNN algorithm the classification can be done by using the MATLAB program. And also for finding the freshness of the fruits we are using $\mathrm{pH}$ analog meter.

\section{WORKING PRINCIPLE OF PROPOSED SYSTEM}

The E-Nose System consists of fruit container, this container having different fruits like pineapple, mango and orange. The gas from fruit container is sent to sensor chamber, the sensors MQ-3 which detects the alcohol gasand MQ-135 sensor which detects the ammonia gas. The sensor detected values are given to the serial monitor of the Arduino .If the gas detected by the sensors are in the range of mentioned values, and then the fruit is classified correctly. The ranges of values are obtained from K-NN algorithm. The flow of work is show in the Fig.2.

In the Fig.2 S1 represents the MQ-3 sensor which detects the alcohol content and S2 represents the MQ-135 sensor which detects the ammonia gas.

\section{i.K-NN Algorithm}

$\mathrm{K}-\mathrm{NN}$ algorithm is a pattern reorganization algorithm, which is used for classification and regression. In above mentioned two cases the input contains $\mathrm{K}$ nearest training samples in the feature slot. The outcome of the K-NN algorithm is depends on the whether it is used as classification purpose or for regression. The output of K-NN classification is a class membership, and a thing is classified by more votes of its neighbors, with the object being assigned to the class most common among its $\mathrm{k}$ nearest neighbours [11]. ( $\mathrm{K}$ is a positive integer, mostly small). If $\mathrm{k}$ $=1$, then the thing is simply given to the class of that single nearest neighbour. 


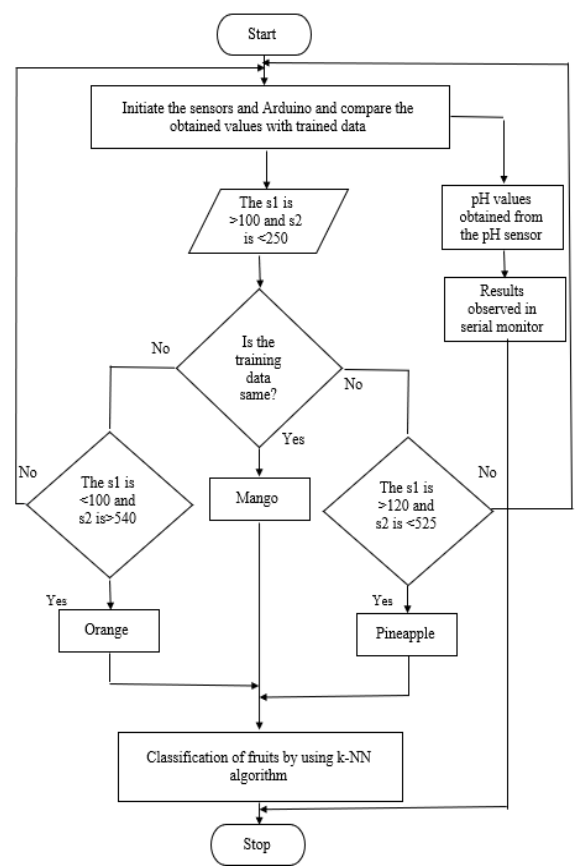

Fig.2. Work Flow of Electronic-Nose System

ii. Pattern Reorganization of Fruits

The pattern recognition of mango in 3D view shown in Fig.3 .pattern is obtained from training data which are collected from mango fruit.

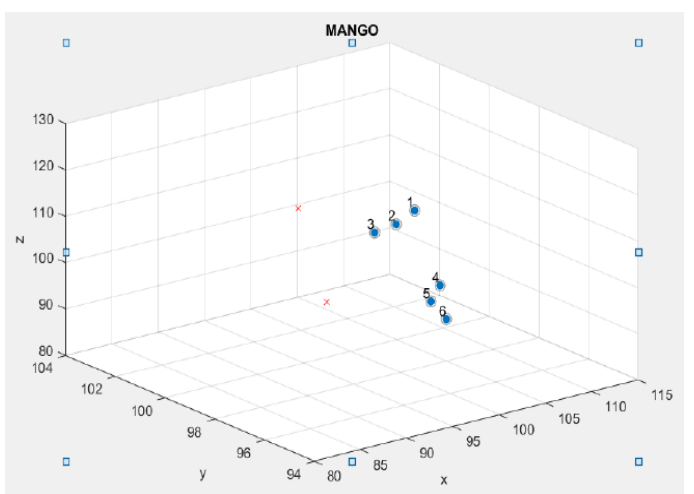

Fig.3. Pattern recognition of mango fruit in $3 \mathrm{D}$ view

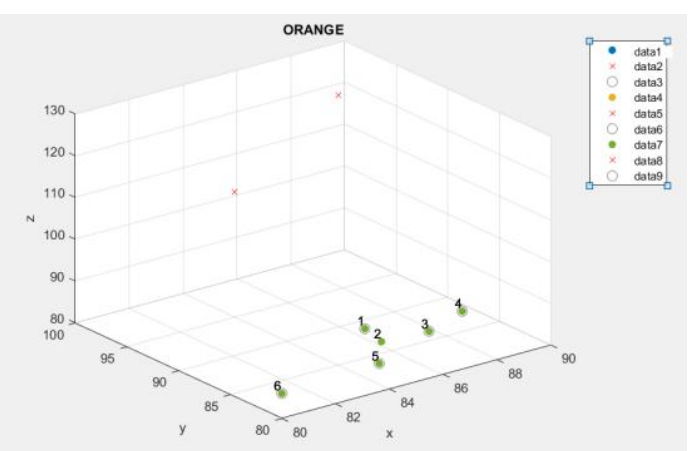

Fig.4. Pattern recognition of orange fruit in $3 D$ view

The pattern recognition of orange and pineapple in 3D view shown in Fig.4 and Fig.5 respectively .pattern is obtained from training data which are collected from orange fruit.

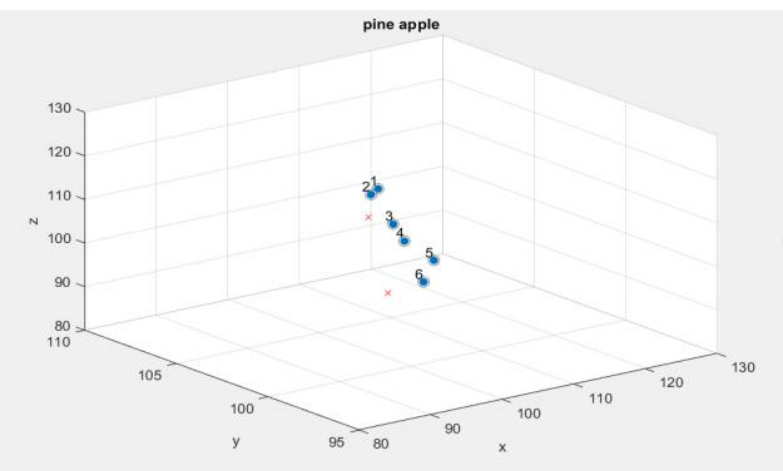

Fig.5. Pattern recognition of pineapple fruit in 3D view

\section{RESULTS}

\section{a. Classification of fruits}

The sensor MQ3 and MQ135 sense the fruit gas which is fed to Arduino controller, this controller compare the generated pattern value with sensor output value and gives the output as the fruit name such as mango, pineapple and orange. The prototype of E-Nose system is shown in the Fig.6.

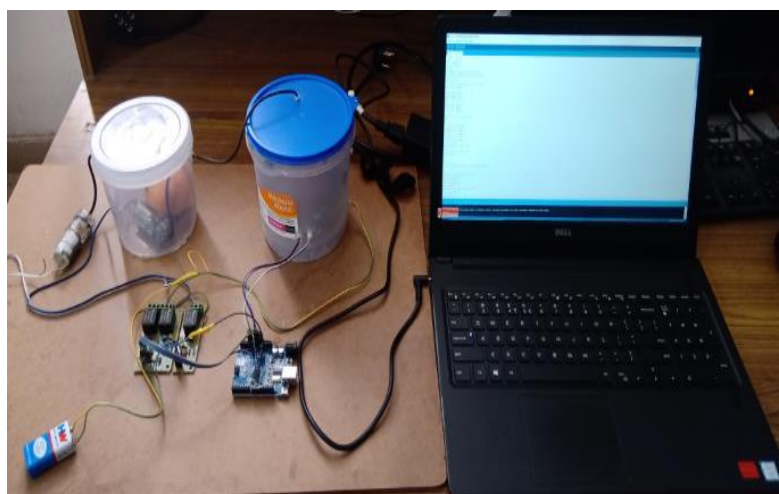

Fig.6.Equipment setup of E-nose for classification of fruits

\section{b. Freshness Measurement}

A pH sensor is included in the sensor chamber by which the $\mathrm{pH}$ of different fruits can be determined (expressed in terms of $\mathrm{pH}$ value) which gives the freshness of the fruit in terms of acidity shown in Fig.7

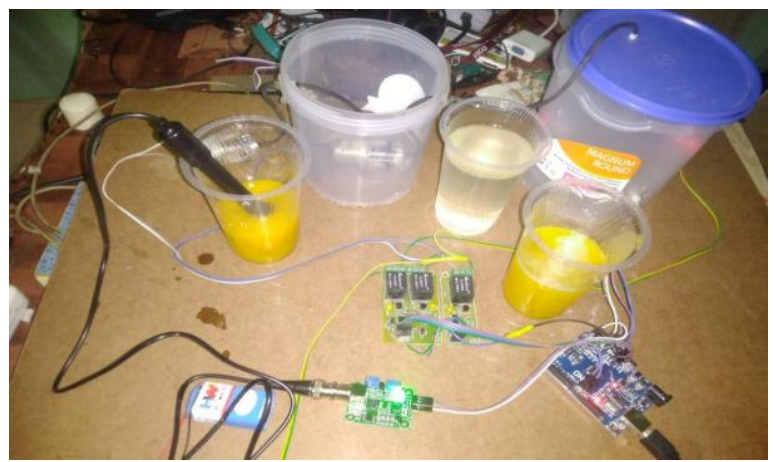

Fig.7 Equipment setup of E-Nose for freshness measurement of fruit juice 


\section{CONCLUSION}

The proposed E-nose which is used to classify the fruits and freshness of a fruit is successfully designed and tested. By integrating all the hardware components along with the MATLAB software the classification of different fruits and freshness of the fruit juice are determined.

\section{REFERENCES}

1. Paul E. Keller Lars J. Kangas, Lars H. Liden, SherifHashem, Richard T. Kouzes "Electronic Noses And Their Applications," 13 December 2010.

2. Assessment of odours from livestock wastes by a photoionization detector, an electronic nose, olfactometry and gas chromatography-mass spectrometry

3. H. Troy Nagle, Susan S. Schiffman and Ricardo GutierrezOsuna, North Carolina State University Duke University Medical Center, "The how and why E-nose" September 2008.

4. .Kea-Tiong Tang, Shih-Wen Chiu, Chih-Heng Pan, Hung-Yi Hsieh, Yao-Sheng Liang and Ssu-Chieh Liu "Development of portable e-nose for the detection of fruity odours," 15 October 2011.

5. http://www.hwsensor.com

6. http://www.hwsensor.com

7. E201-BNC pH Electrode, Bante Instruments, technical manual Rev 1r0.

8. Tropic fruit mango, Maria Cecilia Franco Martínez, Victoria BenítezOlaya, Daniel Gaviria Valenzuela Head of R\&D Quality Control Director Manager, January 28th, 2015.

9. Liquid fruits FRULIX TF ABACAXI pineapple, Rua Cardoso Quintão.

10. Tropic fruit orange, Maria Cecilia Franco Martínez, Victoria BenítezOlaya, Daniel Gaviria Valenzuela Head of R\&D Quality Control Director Manager, January 28th, 2015.

11. H. Wang and D. Bell, "Extended K-Nearest Neighbors based on Evidence Theory”, Computer Journal 47(6): pp 